\title{
Ensuring Adequate Vitamin B12 Status on a Plant-Based Diet
}

\author{
Stewart D Rose* and Amanda J Strombom \\ Plant-Based Diets in Medicine, USA
}

Submission: June 18, 2019; Published: July 09, 2019

*Corresponding author: Stewart Rose, Plant-Based Diets in Medicine, 12819 SE 38th St, \#427, Bellevue, WA 98006, USA

\begin{abstract}
Vitamin B12 is a water-soluble vitamin. All naturally occurring B12 is produced by bacteria in the soil. Animals ingest these bacteria along with their feed, resulting in their presence in animal-derived foods. It is also added to other foods as an enrichment and is available as a dietary supplement. Since patients on a plant-based diet eat no animal-derived foods, and only plant-foods cleaned of soil residue, they must rely on supplementation or fortified foods. Status is typically assessed via serum or plasma vitamin B12 levels. Injected Vitamin B12 appears to be equivalent to oral vitamin B12, but there is no evidence that sublingual delivery offers any advantage over other oral preparations. Vitamin B12 has been demonstrated to be safe in doses up to 1,000 times the recommended dietary allowance and is safe in pregnancy.

It is vital that patients on a plant-based diet have adequate levels of Vitamin B12. Vitamin B12 deficiency is characterized by megaloblastic anemia, fatigue, weakness, constipation, loss of appetite, and weight loss. Neurological changes, such as numbness and tingling in the hands and feet, can also occur. Due to conservation through enterohepatic recirculation, the body's stores of B12 may last the patient some time which can lead to complacency and give the patient a false sense of security. Therefore, the physician should emphasize the importance of vitamin B12 supplementation and the rationale for it.
\end{abstract}

Keywords: B12 deficiency; Lactation; Megaloblastic anemia; Plant-based diet; Pregnancy; Supplementation; Vegan; Vegetarian; Vitamin B12

Abbreviations: IF: Intrinsic Factor; Mcg: Micrograms; IOM: The Institute of Medicine; RDAs: Recommended Dietary Allowances; AI: Adequate Intake

\section{Introduction}

Vitamin B12 is a water-soluble vitamin that is naturally present in animal-derived foods. It is added to others and is available as a dietary supplement and a prescription medication. Vitamin B12 exists in several forms and contains the mineral cobalt [1-4], so compounds with vitamin B12 activity are collectively called "cobalamins". Methylcobalamin and 5-deoxyadenosylcobalamin are the forms of vitamin B12 that are active in human metabolism [5].

Vitamin B12 is required for proper red blood cell formation, neurological function, and DNA synthesis [1-5]. Vitamin B12 functions as a cofactor for methionine synthase and L-methylmalonyl-CoA mutase. Methionine synthase catalyzes the conversion of homocysteine to methionine [5,6]. Methionine is required for the formation of S-adenosylmethionine, a universal methyl donor for almost 100 different substrates, including DNA, RNA, hormones, proteins, and lipids. L-methylmalonyl-CoA mutase converts L-methylmalonyl-CoA to succinyl-CoA in the degradation of propionate $[3,5,6]$, an essential biochemical reaction in fat and protein metabolism. Succinyl-CoA is also required for hemoglobin synthesis. Thus, maintaining adequate vitamin B12 status is vital for good health.

Vitamin B12, bound to protein in animal-derived foods, is released by the activity of hydrochloric acid and gastric protease in the stomach [5]. When synthetic vitamin B12 is added to fortified foods and dietary supplements, it is already in free form and thus does not require this separation step. Free vitamin B12 then combines with intrinsic factor (IF), a glycoprotein secreted by the stomach's parietal cells, and the resulting complex undergoes absorption within the distal ileum by receptor-mediated endocytosis [5,7].

\section{How much is needed?}

Vitamin B12 status is typically assessed via serum or plasma vitamin B12 levels. Values below approximately 170-250 pg/ $\mathrm{mL}$ (120-180 picomol/L) for adults [5] indicate a vitamin B12 deficiency. However, evidence suggests that serum vitamin 
B12 concentrations might not accurately reflect intracellular concentrations [6]. Elevated methylmalonic acid levels (values $>0.4$ micromol/L) might be a more reliable indicator of vitamin B12 status because they indicate a metabolic change that is highly specific to vitamin B12 deficiency [5-9].

The following Table 1 lists the current RDAs for vitamin B12 in micrograms $(\mathrm{mcg})$ [5]. For infants aged 0 to 12 months, the Food and Nutrition Board established an adequate intake (AI) for vitamin B12 that is equivalent to the mean intake of vitamin B12 in healthy, breastfed infants.

Table 1: Recommended Dietary Allowances (RDAs) for Vitamin B12.

\begin{tabular}{|c|c|c|c|}
\hline Life Stage & Age & Males $(\mu \mathrm{g} /$ day) & Females $(\mu \mathrm{g} /$ day) \\
\hline Infants & $0-6$ months & $0.4(\mathrm{AI})$ & $0.4(\mathrm{AI})$ \\
\hline Infants & $7-12$ months & $0.5(\mathrm{AI})$ & $0.5(\mathrm{AI})$ \\
\hline Children & 1-3 years & 0.9 & 0.9 \\
\hline Children & 4-8 years & 1.2 & 1.2 \\
\hline Children & 9-13 years & 1.8 & 1.8 \\
\hline Adolescents & 14-18 years & 2.4 & 2.4 \\
\hline Adults & 19-50 years & 2.4 & 2.4 \\
\hline Adults & $\begin{array}{c}51 \text { years and } \\
\text { older }\end{array}$ & $2.4^{*}$ & $2.4^{*}$ \\
\hline Pregnancy & all ages & - & 2.6 \\
\hline Breast-feeding & all ages & - & 2.8 \\
\hline
\end{tabular}

*Vitamin B12 intake should be from supplements or fortified foods due to the age-related increase in food-bound malabsorption.

Approximately $56 \%$ of a $1 \mathrm{mcg}$ oral dose of vitamin B12 is absorbed, but absorption decreases drastically when the capacity of intrinsic factor is exceeded (at 1-2 mcg of vitamin B12) [10]. After the age of 50, the amount of intrinsic factor available is reduced, leading to malabsorption in many seniors. However, approximately $1 \%$ of oral vitamin B12 can be absorbed passively in the absence of intrinsic factor $[8,9]$. For example, only about 5 mcg of a 500-mcg oral supplement is actually absorbed in healthy people [10].

The bioavailability of sublingual vitamin B12 appears to be equivalent to oral vitamin B12, but there is no evidence that sublingual delivery offers any advantage over oral preparations [11]. Given the lower cost and ease of administration of oral vitamin B12, this might be a reasonable choice for replacement in many patients [12].

Vitamin B12 has been demonstrated to be safe in doses up to 1,000 times the recommended dietary allowance and is safe in pregnancy [5]. The Institute of Medicine (IOM) did not establish an upper limit for vitamin B12 because of its low potential for toxicity. The IOM states that "no adverse effects have been associated with excess vitamin B12 intake from food and supplements in healthy individuals" [5]. Findings from an intervention trial support these conclusions. In the HOPE 2 trial, vitamin B12 supplementation (in combination with folic acid and vitamin B6) did not cause any serious adverse events when administered at doses of $1.0 \mathrm{mg}$ for 5 years [13].

\section{Vitamin B12 for plant-based diet consumers}

All naturally occurring B12 is produced by microorganisms [14]. The only organisms to produce vitamin B12 are certain species of bacteria. Some of these bacteria are found in the soil around the grasses that ruminants eat. They are taken into the animal, proliferate, form part of their gut flora, and continue to produce vitamin B12 [14]. Since patients on a plant-based diet eat no animal-derived foods, and only plant-foods cleaned of soil residue, they must rely on supplementation or fortified foods.

Fortified breakfast cereals are a readily available source of vitamin B12 with high bioavailability for vegetarians $[5,8,9,15,16]$. Some nutritional yeast products also contain vitamin B12. Fortified foods vary in formulation between brands and over time, so it is important to read product labels regularly to determine which added nutrients they contain.

Several studies have shown varying prevalence of B12 deficiency with those on a plant- based diet [17-19]. For instance, one study showed that (52\%) of those following a plant-based diet were B12 deficient ( $<118$ pmol/L) [20]. However, other studies have found a lower incidence of B12 deficiency [21]. As expected, those taking B12 containing supplements have lower incidences of B12 deficiency [20].

Vitamin B12 is secreted in bile and then most is reabsorbed via the enterohepatic circulation by ileal receptors which require IF. In healthy individuals most of this B12 is reabsorbed and available for metabolic functions [5]. This gives the body an effective mechanism to conserve its B12, so deficiency can take as long as several years to present. This sometimes leads to complacency among patients following a plant-based diet. Development of vitamin B12 deficiency is likely to be more rapid in patients with pernicious anemia since IF is lacking [5].

\section{Vitamin B12 deficiency}

Vitamin B12 deficiency is characterized by megaloblastic anemia, fatigue, weakness, constipation, loss of appetite, and weight loss $[2,3,22]$. Neurological changes, such as numbness and tingling in the hands and feet, can also occur [5,23]. Additional symptoms of vitamin B12 deficiency include difficulty maintaining balance, depression, confusion, dementia, poor memory, and soreness of the mouth or tongue [23]. The neurological symptoms of vitamin B12 deficiency can occur without anemia, so early diagnosis and intervention is important to avoid irreversible damage [6].

Large amounts of folic acid can mask the damaging effects of vitamin B12 deficiency by correcting the megaloblastic anemia caused by vitamin B12 deficiency [2,24] without correcting the neurological damage that also occurs [2,24].

Vitamin B12 crosses the placenta during pregnancy and is present in breast milk. Exclusively breastfed infants of women who consume no animal products may have very limited reserves of vitamin B12 and they can develop vitamin B12 deficiency within months of birth $[5,25]$. Undetected and untreated vitamin 
B12 deficiency in infants can result in severe and permanent neurological damage. During infancy, signs of a vitamin B12 deficiency include failure to thrive, movement disorders, developmental delays, and megaloblastic anemia [26].

\section{Treating B12 deficiency}

Vitamin B12 deficiency is sometimes treated with vitamin B12 injections, since this method bypasses potential barriers to absorption. However, high doses of oral vitamin B12 may also be effective. In a 2005 review of randomized controlled trials comparing oral with intramuscular vitamin B12, patients who received high dosages of oral vitamin B12 (1 to $2 \mathrm{mg}$ daily) for 90 to 120 days had an improvement in serum vitamin B12 similar to patients who received intramuscular injections. The authors concluded that 2,000 mcg of oral vitamin B12 daily, followed by a decreased daily dose of 1,000 mcg and then 1,000 mcg weekly, can be as effective as intramuscular administration $[8,9]$. These results were consistent in patients regardless of the etiology of their vitamin B12 deficiency, including malabsorption states and pernicious anemia. Overall, an individual patient's ability to absorb vitamin B12 from the diet is the most important factor in determining whether vitamin B12 should be administered orally or via injection [10].

The American Dietetic Association recommends supplemental vitamin B12 for vegans and lacto-ovo vegetarians during both pregnancy and lactation to ensure that enough vitamin B12 is transferred to the fetus and infant [27]. Pregnant and lactating women who follow strict vegetarian or vegan diets should consult with a pediatrician regarding vitamin B12 supplements for their infants and children [5].

\section{Discussion}

In previous eras and under primitive conditions, man may have absorbed adequate vitamin B12 through the soil left on his food when there was little to no washing and peeling. Now, however, patients following a plant-based, or vegan diet, must supplement with vitamin B12. Due to conservation through enterohepatic recirculation, the body's stores of B12 may last the patient some time.

Some vegetarian and vegan patients are complacent when it comes to vitamin B12 supplementation. The long lag time until symptoms present can add to the complacency and some patients may become convinced, they don't need supplemental B12. Therefore, the physician should emphasize the importance of vitamin B12 supplementation and the rationale for it. The physicians should also make the patient aware of the consequences of deficiency.

Given the higher rate of B12 deficiency in vegans and vegetarians, they should have the lab work done initially. If a deficiency is found, or there is non-compliance with recommended supplementation, then more frequent lab work is called for.

Vitamin B12 deficiency is easy to prevent. Vitamin B12 is included in many multivitamins as well as being sold separately.
While some foods are fortified with B12, this cannot be relied upon due to dietary preference variations and commercial reformulation.

$500 \mathrm{mcg}$ and 1,000 mcg vitamin B12 supplements, as well as smaller doses, are widely available and affordable and can be used to prevent or treat deficiency, regardless of etiology, without the need for intramuscular injections. Considering the exceptionally low toxicity, vitamin B12 can be prescribed in generous doses if the clinician considers it best to err on the side of safety with a patient.

\section{References}

1. Herbert VKD (1994) Vitamin B12 in Modern Nutrition in Health and Disease. ( $8^{\text {th }}$ edn.), Williams \& Wilkins, Baltimore, Maryland, USA.

2. Herbert V (1996) Vitamin B12 in Present Knowledge in Nutrition. (17 ${ }^{\text {th }}$ edn.), International Life Sciences Institute Press, Washington, DC, USA.

3. Combs G (1992) Vitamin B12. The Vitamins: Fundamental aspects in nutrition and health. ( $3^{\text {rd }}$ edn.), Elsevier Academic Press, Burlington, MA, USA.

4. Zittoun J, Zittoun R (1999) Modern clinical testing strategies in cobalamin and folate deficiency. Semin Hematol 36(1): 35-46.

5. (1998) Dietary Reference Intakes for Thiamin, Riboflavin, Niacin, Vitamin B6, Folate, Vitamin B12, Pantothenic Acid, Biotin, and Choline. National Academies Press, Washington, DC, USA.

6. Clarke R (2008) B-vitamins and prevention of dementia. PRoc Nutr Soc 67(1): 75-81.

7. Klee G (2000) Cobalamin and folate evaluation: measurement of methylmalonic acid and homocysteine vs vitamin B (12) and folate. Clin Chem 46(8Pt 2): 1277-1283.

8. Vidal-Alaball J, Butler C, Cannings-John R, Goringe A, Hood K, et al. (2005) Oral vitamin B12 versus intramuscular vitamin B12 for vitamin B12 deficiency. Cochrane Database Syst Rev (3): CD004655.

9. Butler C, Vidal-Alaball J, Cannings-John R, McCaddon A, Hood K, et al. (2006) Oral vitamin B12 versus intramuscular vitamin B12 for vitamin B12 deficiency: a systematic review of randomized controlled trials. Fam Pract 23(3): 279-285.

10. Carmel R (2008) How I treat cobalamin (vitamin B12) deficiency. Blood 112(6): 2214-2221.

11. Sharabi A, Cohen E, Sulkes J, Garty M (2003) Replacement therapy for vitamin B12 deficiency: comparison between the sublingual and oral route. Br J Clin Pharmacol 56(6): 635-638.

12. Langan R, Zawistoski K (2011) Update on Vitamin B12 Deficiency. American Family Physician 83(12): 1425-1430.

13. Lonn E, Yusuf S, Arnold M, Sheridan P, Pogue J, et al. (2006) Homocysteine lowering with folic acid and B vitamins in vascular disease. N Engl J Med 354(15): 1567-1577.

14. Groff J, Gropper S, Hunt S (1995) Advanced nutrition and human metabolism. ( $2^{\text {nd }}$ edn.), West Publishing Comp, Minneapolis.

15. Subar A, Krebs-Smith S, Cook A, Kahle L (1998) Dietary sources of nutrients among US adults, 1989 to 1991. J Am Diet Assoc 98(5): 537547.

16. Tucker K, Rich S, Rosenberg I, Jacques P, Dallal G, et al. (2000) Plasma vitamin B-12 concentrations relate to intake source in the Framingham Offspring study. Am J Clin Nutr 71(2): 514-522.

17. Wokes F, Badenoch J, Sinclair H (1955) Human dietary deficiency of vitamin B12. Am J Clin Nutr 3(5): 375-382. 
18. Herrmann W, Schorr H, Purschwitz K, Rassoul F, Richter V (2001) Total homocysteine, vitamin B (12) and total antioxidant status in vegetarians. Clin Chem 47(6): 1094-1101.

19. Herrmann W, Schorr H, Obeid R, Geisel J (2003) Vitamin B-12 status, particularly holotranscobalamin II and methylmalonic acid concentrations, and hyperhomocysteinemia in vegetarians. Am J Clin Nutr 78(1): 131-136.

20. Gilsing A, Crowe F, Lloyd-Wright Z, Sanders T, Appleby P, et al. (2010) Serum concentrations of vitamin B12 and folate in British male omnivores, vegetarians and vegans: results from a cross-sectional analysis of the EPIC-Oxford cohort study. Eur J Clin Nutr 64(9): 933939.

21. Majchrzak D, Singer I, Männer M, Rust P, Genser D, et al. (2006) B-vitamin status and concentrations of homocysteine in Austrian omnivores, vegetarians and vegans. Ann Nutr Metab 50(6): 485-491.

22. Bernard M, Nakonezny P, Kashner T (1998) The effect of vitamin B12 deficiency on older veterans and its relationship to health. J Am Geriatr Soc 46(10): 1199-1206.
23. Bottiglieri T (1996) Folate, vitamin B12, and neuropsychiatric disorders. Nutr Rev 54(12): 382-390.

24. Chanarin I (1994) Adverse effects of increased dietary folate. Relation to measures to reduce the incidence of neural tube defects. Clin Invest Med 17(3): 244-252.

25. von Schenck U, Bender-Gotze C, Koletzko B (1997) Persistence of neurological damage induced by dietary vitamin B12 deficiency in infancy. Arch Dis Childhood 77(2): 137-139.

26. Bjørke Monsen A, Ueland P (2003) Homocysteine and methylmalonic acid in diagnosis and risk assessment from infancy to adolescence. Am J Clin Nutr 78(1): 7-21.

27. Kaiser L, Allen L (2008) Position of the American Dietetic Association: nutrition and lifestyle for a healthy pregnancy outcome. J Am Diet Assoc 108(3): 553-561.

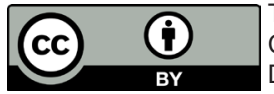

his work is licensed under Creative Commons Attribution 4.0 License DOI: 10.19080/ARGH.2019.13.555862 